\title{
Markers for Identifying and Targeting Glioblastoma Cells during Surgery
}

\author{
Stephanie Schipmann ${ }^{10} \quad$ Michael Schwake $^{1} \quad$ Eric Suero Molina ${ }^{1} \quad$ Walter Stummer $^{2}$ \\ ${ }^{1}$ Department of Neurosurgery, University Hospital Münster, Münster, \\ Germany \\ 2 NCH, UK Münster, Münster, Germany \\ Address for correspondence Stephanie Schipmann, MD, Department \\ of Neurosurgery, University Hospital Münster, Albert-Schweitzer- \\ Campus 1, Building A1, 48149 Münster, Germany \\ (e-mail: Stephanie.schipmann@ukmuenster.de).
}

J Neurol Surg A 2019;80:475-487.

\author{
Abstract \\ Keywords \\ - glioma \\ - interoperative MRI \\ - 5-ALA \\ - fluorescein \\ - indocyanine green
}

Glioblastoma is a highly malignant tumor with a poor prognosis. A factor influencing survival that can be affected by the surgeon is the extent of resection (EOR). Due to the infiltrative nature of the tumor, delineation of tumor from normal brain parenchyma is often challenging. To improve EOR and facilitate tumor visualization, several techniques have been developed over the last few years. This literature review presents an overview of current intraoperative strategies for identifying and targeting glioma cells and discusses the benefits and limitations of each technique. Along with conventional techniques such as neuronavigation and ultrasound, fluorescence-guided surgery with different fluorescent agents such as 5 -aminolevulinc acid and fluorescein have been widely used. Recently, newer techniques have emerged and are being translated into the operating room, promising delineation of glioblastoma tissue using targeted approaches or identification on a microscopic level, for instance using Raman spectroscopy or confocal microscopy.

\section{Introduction}

Glioblastoma is one of the most malignant tumors of the central nervous system. Despite a better understanding of tumor biology and the development of new treatment approaches during the last decades, the tumor remains incurable with a poor prognosis, characterized by a median survival of 15 months and a 2-year survival rate of $17.4 \%{ }^{1,2}$

Current first-line treatment consists of surgical resection, followed by adjuvant radiation and chemotherapy. ${ }^{3}$ Extent of resection (EOR) is an independent predictor of survival, first shown by Lacroix et al, who demonstrated that an EOR $>98 \%$ of tumor volume results in a significant survival advantage. ${ }^{4}$ Another study indicated that the threshold might even be lower, with an EOR of $78 \%$ as a minimum associated with a survival benefit. ${ }^{5}$ In addition, data from a large EORTC-NCIC trial, the pivotal trial for approval of temozolomide as first-line therapy for glioblastoma, revealed a greater benefit from adjuvant radiotherapy and chemotherapy in patients with gross total resection (GTR). ${ }^{6}$

Clearly, EOR is a critical driver of outcome in glioblastoma and the only factor that the surgeon can influence directly. Given the infiltrative nature and often eloquent tumor localization, achieving the largest possible EOR is often challenging. Tumor infiltration into the surrounding brain parenchyma is difficult to discriminate with the human eye under standard white light microscopy, and differentiation of tumor margins from normal brain often cannot be performed based on tactile features of tissues. Consequently, several techniques have been developed to improve the ability of the surgeon to identity glioblastoma tissue during surgery.

Several modalities aiming at improving EOR entered the field decades ago, such as neuronavigation, intraoperative ultrasound, and intraoperative magnetic resonance imaging (iMRI). Newer techniques, such as fluorescence-guided surgery received

December 30, 2018

accepted after revision

March 4, 2019

published online

August 29, 2019 $\begin{array}{ll}\text { ๑) } 2019 \text { Georg Thieme Verlag KG } & \text { DOI https://doi.org/ } \\ \text { Stuttgart · New York } & 10.1055 / \mathrm{s}-0039-1692976 . \\ & \text { ISSN 2193-6315. }\end{array}$ 
(FGS) using various fluorescent agents, enables intraoperative real-time imaging of glioblastoma. Recently, more novel techniques have emerged and are being translated into the operating room, promising an even better delineation of glioblastoma tissue, such as Raman spectroscopy, confocal microscopy, or methods of targeted fluorescence. ${ }^{7-11}$

We performed a literature review on currently available techniques that aim at targeting and identifying glioblastoma cells during surgery, discussing their benefits, applicability, and limitations in the field of glioma surgery. This review focuses on FGS and newer techniques; conventional techniques are only mentioned for the sake of completeness but are not reviewed in further detail.

\section{Conventional Techniques for Intraoperative Visualization of Glioblastoma}

For most surgical glioblastoma cases, conventional techniques such as neuronavigation and ultrasound are standard of care and have been widely integrated into the operative setting.

\section{Neuronavigation and Ultrasound}

Neuronavigation is a basic and ubiquitously available tool for glioblastoma surgery. All relevant preoperative digital scans such as computed tomography (CT), MRI, and positron emission tomography (PET) can be incorporated into the data set for the navigational system and help the surgeon maintain a precise sense of complex three-dimensional anatomical relationships and almost real-time intraoperative localization. ${ }^{12}$ Neuronavigation benefits from high surgical accuracy for resection of glioblastomas and can aid in planning the surgical approach. ${ }^{13,14}$ A study by Wirtz et al evaluated the effect of neuronavigation on the EOR in glioblastoma compared with standard use of the microscope. They showed that the amount of residual tumor was significant lower in the patients operated on using neuronavigation, without showing a clear difference regarding the number of radical resections. ${ }^{15} \mathrm{~A}$ major limitation using neuronavigation is the loss of accuracy caused by intraoperative brain shift due to application of mannitol, drainage of cerebrospinal fluid, patient positioning, and resection of tissue. ${ }^{16}$

Another uncomplicated and cost-effective method for intraoperative glioblastoma localization is ultrasound. This dynamic method helps identify tumor borders and normal brain structures. ${ }^{16}$ A retrospective analysis showed an increase of survival when surgeons used intraoperative ultrasound for identifying residual glioma. ${ }^{17}$ However, concerns have been expressed regarding a sometimes poor differentiation of tumor from the zone of peritumoral edema, putting patients at risk for too extensive resections with neurologic sequelae. ${ }^{18}$ In addition, ultrasound has its limitations in the delineation of normal brain tissue from high-grade glioma tumor borders after previous irradiation. ${ }^{19}$

Intraoperative acquired data from ultrasound can be used to update the navigation system and help overcome the limitation of brain shift. ${ }^{20}$

\section{Intraoperative MRI}

First introduced in the 1990s, iMRI has since undergone further development. It provides almost real-time images during surgery for identification of residual tumor and can also be used to detect possible intraoperative complications such as hematoma. ${ }^{21}$

In addition, the acquired images can be used to update the neuronavigational system to compensate impaired accuracy from brain shift. ${ }^{22,23}$ A prospective randomized controlled trial published by Senft et al compared the rates of GTR in glioma patients operated using conventional microsurgery and patients in whom iMRI was used. These authors showed a significant higher frequency of GTR in the iMRI group (96\% iMRI group versus $68 \%$ control group; $p=0.023$ ), providing evidence for the beneficial role of iMRI in glioma surgery. ${ }^{24}$ Supporting results were presented by Hatiboglu et al, showing that when used by surgeons, iMRI led to an increase of the median EOR from $84 \%$ to $99 \%$ ( $p<0.001$ ) with additional tumor removal after iMRI in contrast-enhancing gliomas. ${ }^{25}$

However, iMRI has distinct disadvantages. This method is expensive, time consuming, and extends the duration of surgery and anesthesia. In addition, repeated application of gadolinium may result in extravasation into the tumor area and resection cavity, leading to false-positive effects. ${ }^{21,26}$

\section{Fluorescence-guided Surgery for Glioblastoma}

The application of fluorescing compounds for differentiating tissue during brain surgery was first described in 1947 by George E. Moore, who noted a higher concentration of fluorescein in brain invaded by malignant glioma using a wood lamp. ${ }^{27}$ After decades of dormancy, this approach was modernized in 1998 by the senior author of this review, Walter Stummer, by the introduction of 5-aminolevulinic acid (5-ALA) for FGS. ${ }^{28}$ FGS is based on the administration of optical imaging agents to patients before surgery, leading to a (selective) accumulation in tumor cells, helpful in intraoperative real-time detection and delineation of tumor tissue. Currently, two agents are being used clinically in the field of glioblastoma surgery: 5-ALA ${ }^{28}$ and fluorescein. ${ }^{27}$ A third dye, indocyanine green (ICG), is under investigation. ${ }^{29}$

\section{5-aminolevulinc Acid}

5-ALA is worldwide the most intensely studied fluorescent agent for brain tumor surgery. It was approved by the European Medicines Agency (EMA) and the U.S. Food and Drug Administration (FDA) for intraoperative visualization of malignant glioma. 5-ALA is a natural metabolite in the hemoglobin pathway and within glioma cells. ${ }^{30,31}$ After oral administration of $20 \mathrm{mg} / \mathrm{kg}$ body weight (BW) 5-ALA (Gliolan in the European Union [Specialized Therapeutics, Melbourne, Australia]; Gleolan in the United States [NX Development Corp., Lexington, Kentucky, United States) 3 hours before the induction of anesthesia, fluorescence can be visualized by using a surgical microscope with a 
xenon light source that can switch between white and violetblue light (wavelength: $370-440 \mathrm{~nm}$ ) and is provided with an emission filter for visualization of red tumor fluorescence with peaks at between 635 and $704 \mathrm{~nm}$, thus well in the red range. ${ }^{30,31}$ Peak fluorescence can be expected after 6 to 8 hours. $^{32,33}$

It was shown that 5-ALA has a high toxicologic safety with only minor side effects such as a temporary and mild elevation of liver enzymes and transient skin phototoxicity. ${ }^{34,35}$

\section{Visualization of Glioblastomas and Intensity of 5-ALA Fluorescence}

The efficacy of 5-ALA for intraoperative visualization of glioblastoma cells was shown by several studies, and investigators uniformly report a high selectivity. In a series of 10 patients, with 89 tissue biopsies, sensitivity of 5-ALA-induced fluorescence for detection of malignant glioma cells was $85 \%$ and specificity was $100 \%{ }^{28}$ In a meta-analysis, including eight studies on histopathologic analysis and intraoperative 5-ALA fluorescence with $>800$ samples from malignant glioma, the specificity for glioblastoma was $88.8 \%$ and sensitivity $82.6 \%{ }^{36}$

Using 5-ALA, it is important to differentiate between different qualities of fluorescence because tumor fluorescence is not homogeneous. Two fluorescence qualities can be distinguished: a vivid solid red fluorescence, representing viable tumor, and a vague, less vivid pink fluorescence, indicating the tumor-infiltrating zone. These findings were supported by histologic and spectroscopic analyses (- Fig. 1). ${ }^{37,38}$

Especially in case of solid fluorescence, a positive predictive value (PPV) of $100 \%$ was reported. PPV was lower, between $91 \%$ and $97 \%$, in tissue with vague fluorescence in invasive areas at the tumor border. ${ }^{38,39}$

Even in recurrent glioblastoma, where tissue scarring and changes induced by previous radiotherapy and chemotherapy are present, 5-ALA-guided resection was still shown to be effective with a PPV of $99.5 \% .{ }^{40}$

Similar findings were reported by Lau et al, who analyzed 211 intraoperative high-grade glioma biopsies from different areas of fluorescence intensity graded from 0 to 3 . They
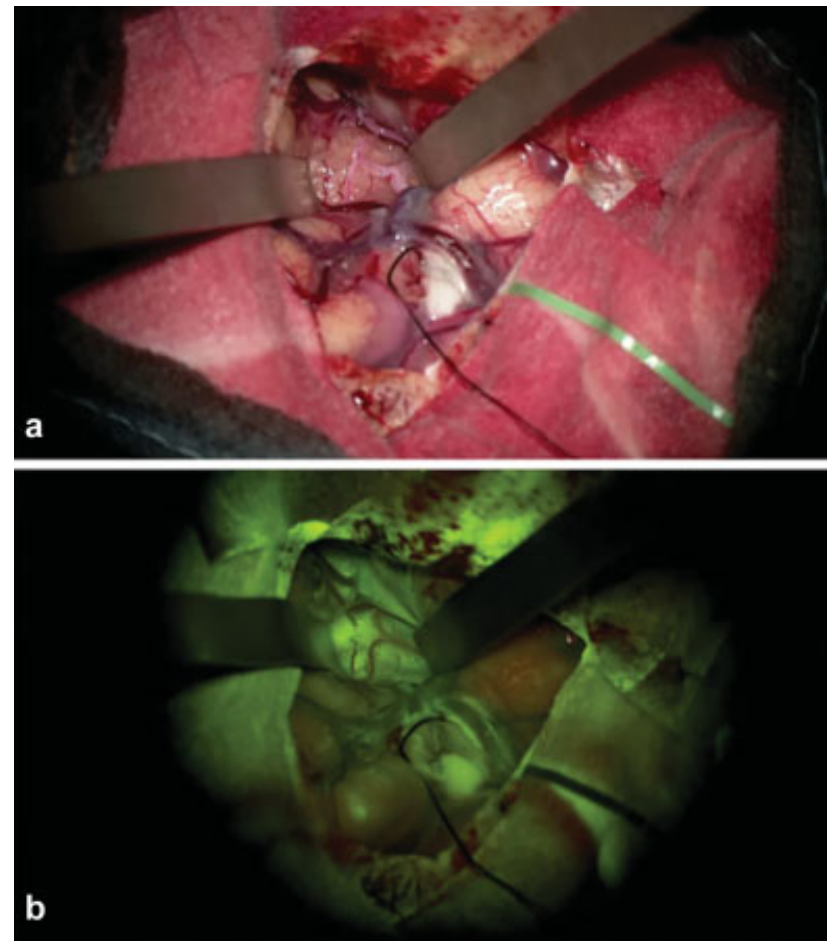

Fig. 2 Use of fluorescein (FL) for resection of glioblastoma. (a) After administration of FL under white light: no fluorescent effect. (b) After administration of FL under YELLOW $560 \mathrm{~nm}$ filter: visible fluorescent effect in the tumor. (Reproduced with permission from Schebesch et al. ${ }^{54}$ )

revealed a PPV of 100\% for high-grade gliomas and $97.2 \%$ for glioblastomas in case of highest rated amount of fluorescence. However, the negative predictive value was comparably very low with $16.7 \%$ for high-grade tumors and $43.9 \%$ for glioblastoma, indicating that not all tumor-infiltrated areas may synthesize the dye in concentrations that can be visualized using the surgical microscope. ${ }^{41}$ Consequently, 5-ALA is a very useful marker for tumor cellularity, especially in areas with solid and bright fluorescence.
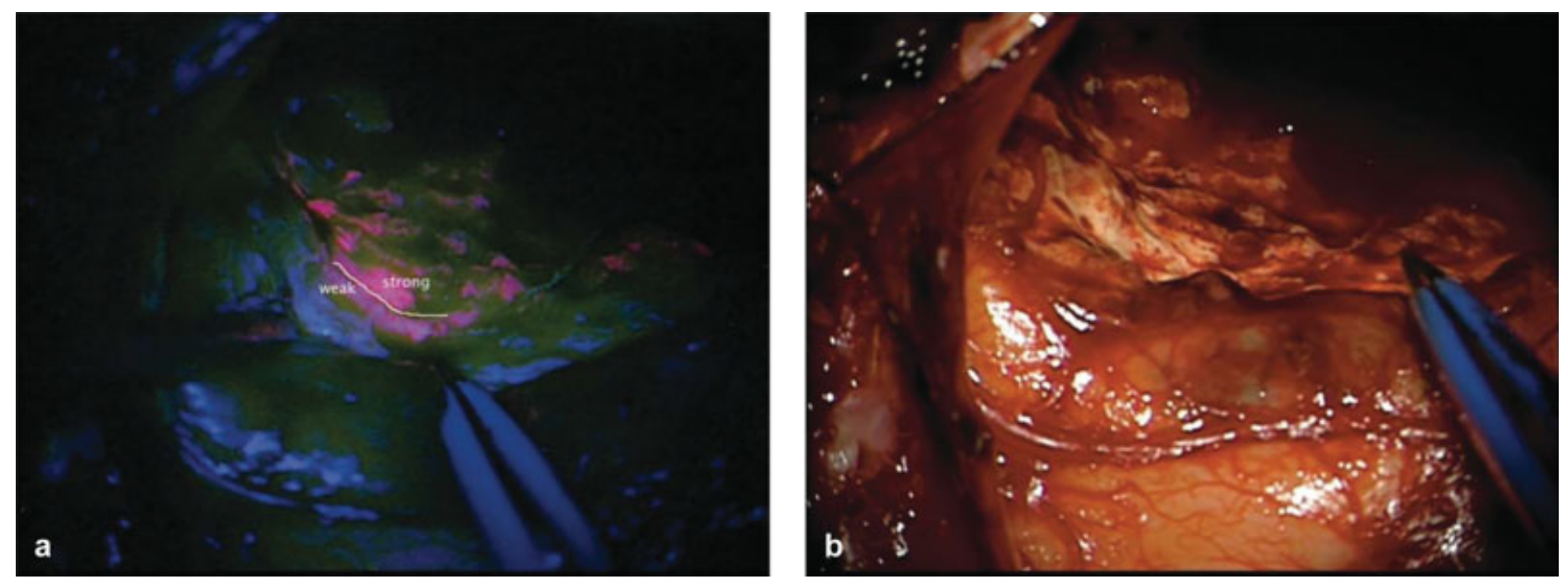

Fig. 1 5-aminolevulinic fluorescence and different fluorescence qualities. (a) Cavity with area of strong (red) and weak (pink) and no fluorescence. (b) Corresponding white light image. (Reproduced with permission from Stummer et al. ${ }^{38}$ ) 
5-ALA has the potential to be used as a tool for detection of solid tumor that can be removed without risk of neurologic deficit but will also help discrimination of infiltrated brain down to a tumor cell density of $\sim 10$ to $20 \%$, enabling even larger resection volumes in noneloquent regions than those identified by contrast enhancement on MRI. ${ }^{39}$

\section{Influence of 5-ALA on Extent of Resection and Outcome} The first prospective study evaluating the impact of 5-ALA on the EOR was published in 2000 by Stummer et al, showing that complete resection of contrast-enhancing tumor on MRI was archived in 33 (63\%) of 52 patients. In most of the remaining patients, complete resection could not be performed due to concerns about neurologic safety. In addition, the improved survival was related to the completeness of resection. ${ }^{37} \mathrm{~A}$ large phase III randomized controlled study included 322 patients with suspected malignant glioma who were randomly assigned to 5-ALAguided or conventional microsurgical resection. Complete resection of contrast-enhancing tumor was achieved in 90 of 139 (65\%) patients in the 5-ALA group compared with 47 of $131(36 \%)$ in the conventional group $(p<0.001)$. Furthermore, patients from the 5-ALA group had a longer 6-month progression-free survival (PFS) (41\% versus $21.1 \%$ in the control group; $p=0.0003$ ) with a median PFS of 5.1 months. ${ }^{34}$

Further studies confirmed the benefit of 5-ALA regarding EOR, and since then 5-ALA has been widely used in the resection of glioblastoma. The initially reported resection rate of $65 \%$ that was achieved using 5-ALA FGR was further improved over the last few years due to confidence in the use of the method, as well as advances in intraoperative monitoring and mapping, the latter allowing safe resections in eloquent areas. Díez et al reported GTR rates of $83.3 \%$ (30 of 36 glioblastoma patients), ${ }^{39}$ whereas a retrospective study by Schucht et al reported a GTR of $96 \%$ (51 of 53 patients). ${ }^{42}$ In comparison, GTR under white light microscopy is only achieved in $36 \%$ of patients, ${ }^{34}$ indicating a major benefit of 5-ALA as an intraoperative adjunct for optimizing resection. Even in eloquent areas, the use of 5-ALA FGS, combined with intraoperative mapping or awake surgery, enables GTR rates of up to $76 \%{ }^{43,44}$

A retrospective analysis of 52 glioblastoma patients with optimal resections according to conventional criteria (i.e., complete resection of contrast-enhancing tumors on early postoperative MRI) compared cases with residual fluorescent tissue and complete removal of fluorescent tissue, demonstrating an improved median overall survival (OS) of 27 months (95\% confidence interval [CI], 22.4-31.6) in patients without residual fluorescence compared with 17.5 months (95\% CI, 12.5-22.5) with residual fluorescence. $^{45}$ It is well known that intraoperative fluorescence exceeds the contrast enhancement visible on MRI, by far, marking almost double the resection volume outlined by contrast enhancement on MRI. ${ }^{46}$ These data again underline the potential of 5-ALA for greater EOR and increased survival.

\section{Combination of 5-ALA FGS with Intraoperative MRI}

Coburger et al evaluated the benefit of the additional use of 5-ALA to iMRI in resection of glioblastoma in a prospective cohort and demonstrated that GTR was achieved significantly $(p<0.01)$ more often using the combined approach of 5-ALA and iMRI (100\%) compared with iMRI alone (82\%), with higher mean EOR in the combined group (99.7\% versus $97.4 \%$, respectively; $p<0.004$ ), indicating a synergistic effect of both methods. ${ }^{47}$

Several studies compared the diagnostic accuracy of 5-ALA and iMRI for identifying brain infiltrated by glioma. Coburger et al described a significantly higher sensitivity ( $91 \%$ versus $66 \%$ ) and specificity (90\% versus $60 \%$ ) for detection of malignant glioma than iMRI at the tumor border. ${ }^{48}$ However, so far no clinical evidence has demonstrated the superiority of one method over the other. An ongoing trial (Impact of iMRI on the Extent of Resection in Patients with Newly Diagnosed Glioblastomas: A Prospective Multicenter Parallel Group Clinical Trial [NCT02379572]) aims at providing more data on comparison of both techniques regarding EOR. However, such comparisons may be purely academic because during surgery, technologies should be combined and synergisms utilized in the best interest of patients.

Analyzing the current literature regarding safety and side effects linked to 5-ALA, our data analysis indicates only minor toxicity such as mild and transient erythema or a mild elevation of liver enzymes in single cases without clinically relevant hepatic disorders. Overall, 5-ALA can be considered a very safe and well-tolerated drug.- Table 1 presents an overview of all included studies.

\section{Fluorescein}

Fluorescein sodium, originally and still widely used in ophthalmic surgery for retinal angiography, was introduced into the field of neurosurgery by George E. Moore in 1947, and it was shown to highlight areas of blood-brain barrier (BBB) disruption linked to tumor growth after intravenous application..$^{27,49}$ Fluorescein has a characteristic yellow-green fluorescence, with a peak absorption between 465 and $480 \mathrm{~nm}$ and an emission peak at 500 to $530 \mathrm{~nm}$ (i.e., in the yellow/green range). When administered in high concentrations, fluorescein fluorescence can even be observed under white light. ${ }^{50}$ Fluorescein is considered a safe, robust, and inexpensive fluorophore. In some cases, it leads to transient discoloration of urine and skin after administration, and anaphylactic reactions have been described in a few cases.

However, no severe adverse events have been described using the recommended dosage of 3 to $5 \mathrm{mg} / \mathrm{kg}$ BW. Fluorescein is administered intravenously just after induction of anesthesia. ${ }^{51-53}$ It is distributed via the bloodstream and then extravasates through the disrupted $\mathrm{BBB}$, highlighting regions of the brain with abnormal vasculature, neovascularization, or increased vascular permeability. ${ }^{27,54}$ In malignant gliomas that are characterized by a disruption of the BBB, fluorescein accumulates in the extracellular space of the tumor tissue. In 


\begin{tabular}{|c|c|c|c|c|c|c|c|c|}
\hline 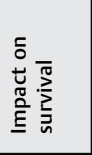 & $\frac{\alpha}{z}$ & $\frac{\alpha}{z}$ & 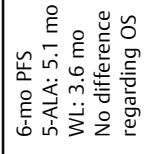 & $\frac{o}{z}$ & $\frac{\alpha}{z}$ & $\frac{\alpha}{z}$ & $\frac{o}{z}$ & $\underline{x}$ \\
\hline$\frac{a_{2}}{2}$ & $\stackrel{\alpha}{z}$ & $\frac{\alpha}{z}$ & $\frac{o}{z}$ & $\frac{\alpha}{z}$ & ఏ̊̊̆ & $\frac{o}{2}$ & $\frac{\alpha}{z}$ & 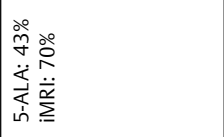 \\
\hline à & ڤั & $\frac{\alpha}{z}$ & $\frac{o}{z}$ & $\stackrel{o}{z}$ & 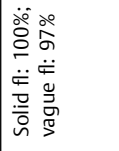 & $\frac{\alpha}{z}$ & $\stackrel{o}{z}$ & 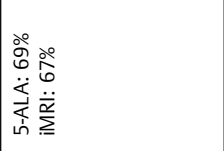 \\
\hline 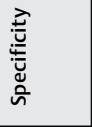 & 。̊ & $\frac{\alpha}{z}$ & $\frac{x}{z}$ & $\frac{\alpha}{z}$ & $\frac{o}{z}$ & $\frac{o}{z}$ & $\frac{\alpha}{z}$ & 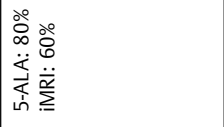 \\
\hline 竧 & $\stackrel{\circ}{\circ}$ & $\underline{p}$ & $\stackrel{\sim}{z}$ & $\stackrel{o}{z}$ & $\stackrel{o}{z}$ & $\stackrel{\frac{\alpha}{z}}{2}$ & $\stackrel{o}{z}$ & 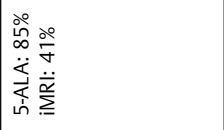 \\
\hline ث̃ & ஓ̊ & ڤે & ڤ్̊ & $\begin{array}{l}\stackrel{\circ}{\circ} \\
\stackrel{\circ}{\sigma}\end{array}$ & 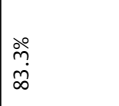 & 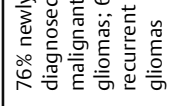 & ळু & $\frac{\alpha}{z}$ \\
\hline 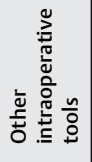 & 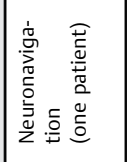 & 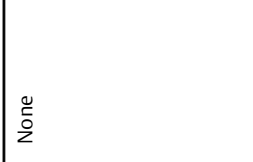 & 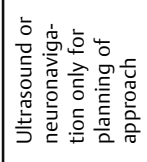 & $\frac{\alpha}{z}$ & 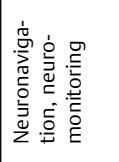 & 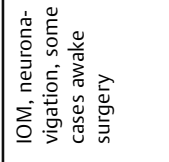 & 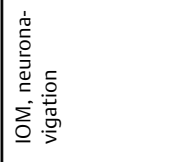 & 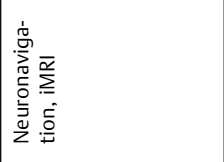 \\
\hline 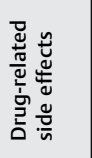 & 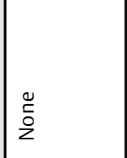 & 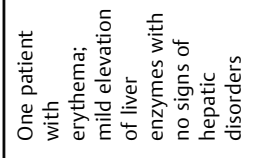 & 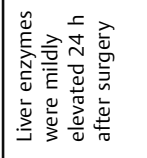 & 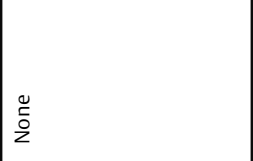 & $\begin{array}{l}\stackrel{0}{\tilde{o}} \\
\frac{1}{2}\end{array}$ & $\begin{array}{l}\stackrel{a}{\tilde{o}} \\
\stackrel{5}{z}\end{array}$ & $\begin{array}{l}\stackrel{0}{\tilde{o}} \\
\stackrel{2}{2}\end{array}$ & 总 \\
\hline 焉 & 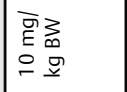 & 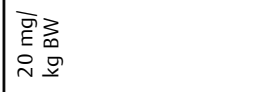 & 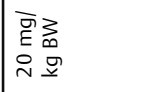 & 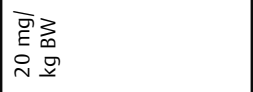 & 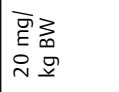 & 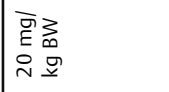 & 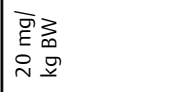 & 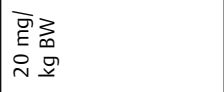 \\
\hline 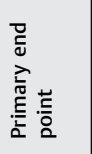 & 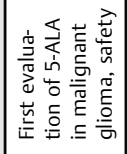 & 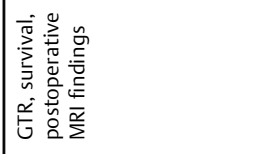 & 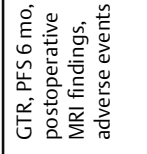 & 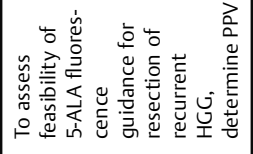 & 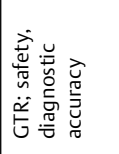 & 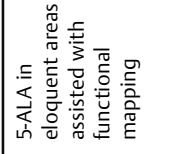 & 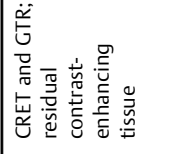 & 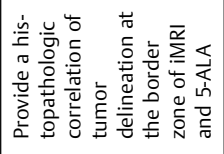 \\
\hline 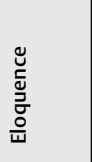 & 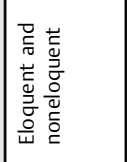 & 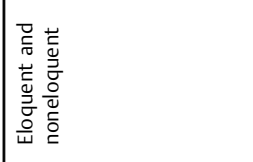 & 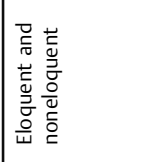 & 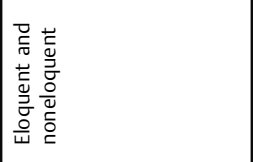 & 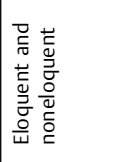 & 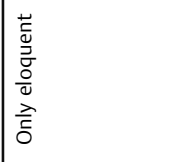 & 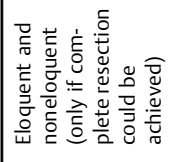 & 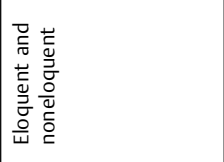 \\
\hline 衰号 & 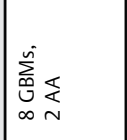 & $\sum_{\mathcal{O}}$ & 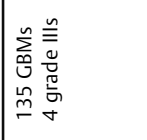 & 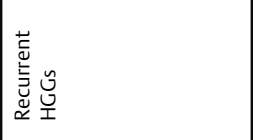 & 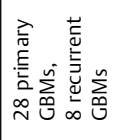 & 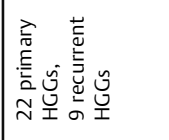 & $\sum_{\mathcal{O}}^{\Sigma}$ & 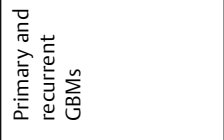 \\
\hline 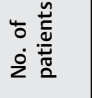 & $\stackrel{\circ}{\circ}$ & ๓ & 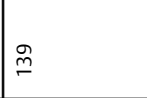 & i & 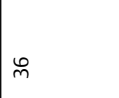 & $\bar{m}$ & $\stackrel{m}{m}$ & 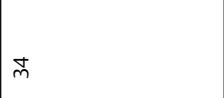 \\
\hline 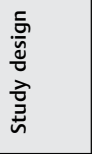 & 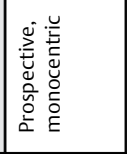 & 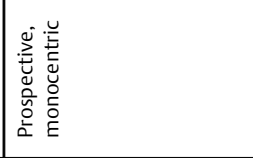 & 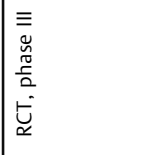 & 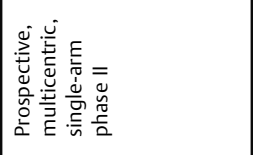 & 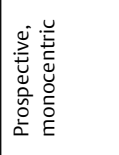 & 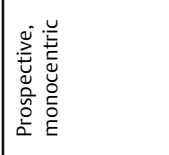 & 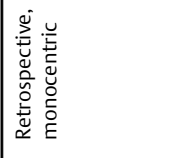 & 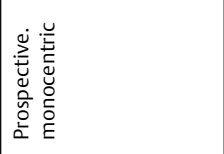 \\
\hline 离 & 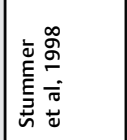 & 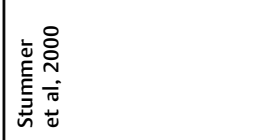 & 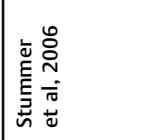 & 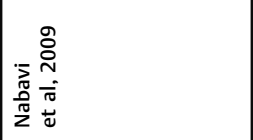 & 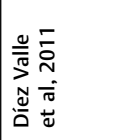 & 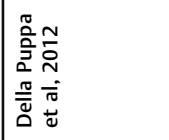 & 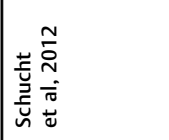 & 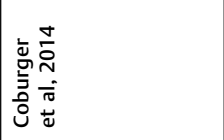 \\
\hline
\end{tabular}




\begin{tabular}{|c|c|c|c|c|c|}
\hline 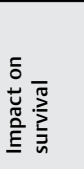 & $\frac{\alpha}{z}$ & $\stackrel{\alpha}{z}$ & 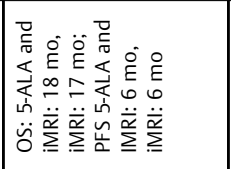 & $\frac{\ddot{z}}{2}$ & 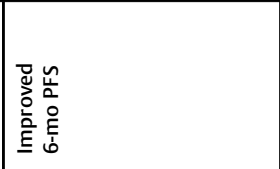 \\
\hline$\frac{\overrightarrow{2}}{2}$ & 岗 & $\stackrel{z}{z}$ & $\stackrel{p}{z}$ & 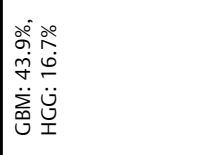 & 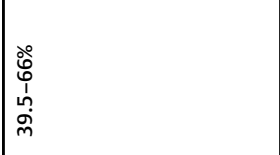 \\
\hline à & 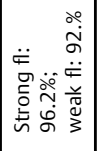 & $\frac{\infty}{z}$ & $\stackrel{p}{z}$ & 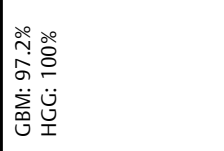 & ڤ̆ \\
\hline 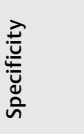 & $\stackrel{\mathscr{z}}{\mathrm{z}}$ & $\ddot{z}$ & $\stackrel{\alpha}{z}$ & 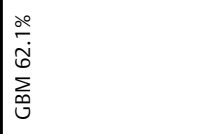 & 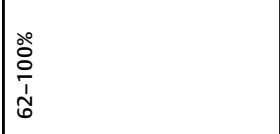 \\
\hline 景 & $\frac{o}{z}$ & $\frac{\mathscr{a}}{z}$ & $\underline{z}$ & 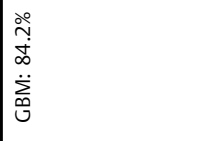 & $\begin{array}{c}\stackrel{0}{0} \\
\infty \\
2\end{array}$ \\
\hline Ë & $\stackrel{p}{z}$ & 登 & 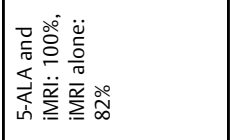 & $\stackrel{x}{z}$ & 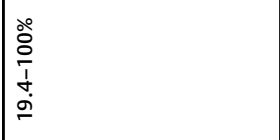 \\
\hline 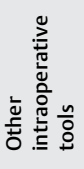 & 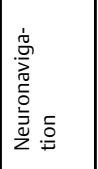 & 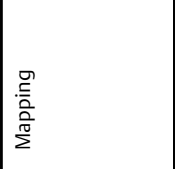 & $\stackrel{\alpha}{z}$ & $\begin{array}{l}\frac{0}{2} \\
\frac{2}{2}\end{array}$ & 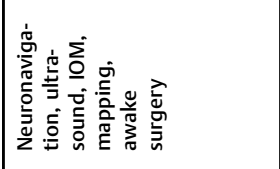 \\
\hline 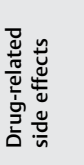 & 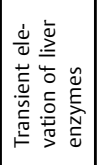 & $\frac{a}{2}$ & 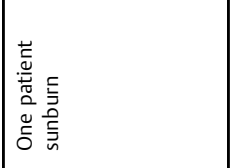 & 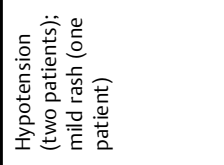 & 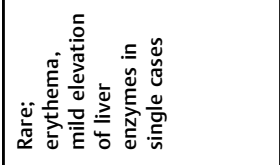 \\
\hline 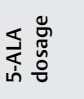 & 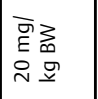 & 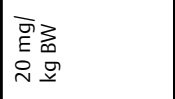 & 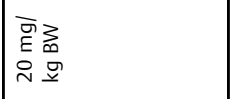 & 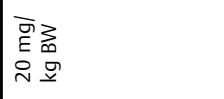 & 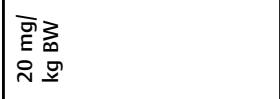 \\
\hline 总 & 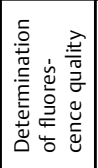 & 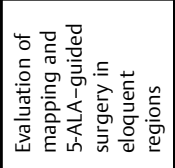 & 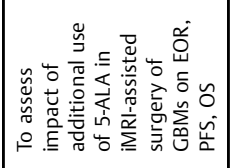 & 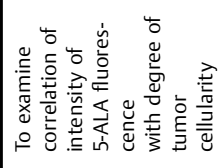 & 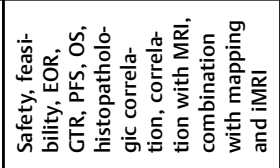 \\
\hline 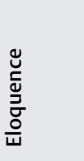 & 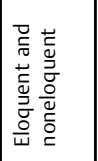 & 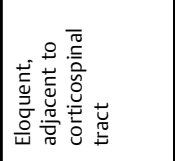 & 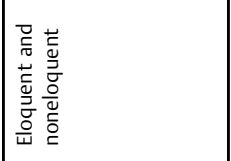 & 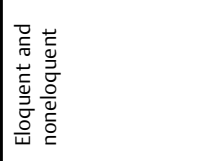 & 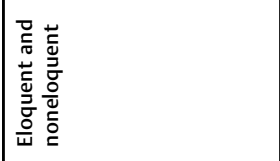 \\
\hline 竧号 & 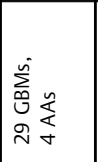 & 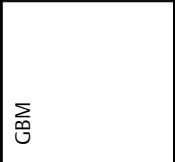 & 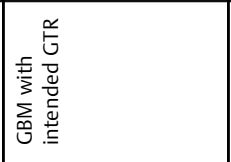 & 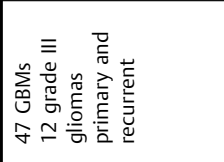 & 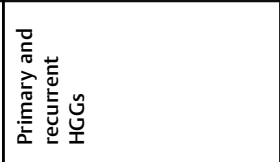 \\
\hline 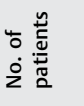 & $\stackrel{m}{m}$ & $\widehat{\iota}$ & m & 옴 & 苛 \\
\hline 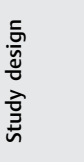 & 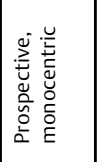 & 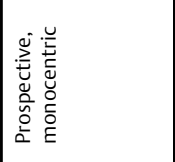 & 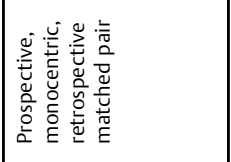 & 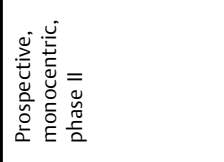 & 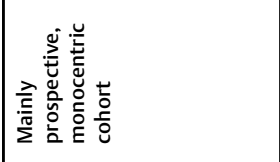 \\
\hline 离 & 童离 & 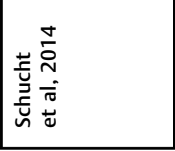 & 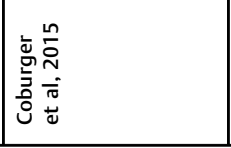 & 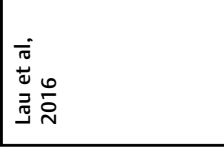 & 离 \\
\hline
\end{tabular}


1998, Kuroiwa et al introduced an operative microscope equipped with emission filters to visualize fluorescein under yellow-filtered $\left(560 \mathrm{~nm}\right.$ ) light. ${ }^{55}$ Today, various fluorescent filters for visualizing fluorescence are available and incorporated into modern surgical microscopes (e.g., the FL560 System [Leica Microscopes, Wetzlar, Germany] and YELLOW 560 system [Carl Zeiss, Dublin, California, United States]) ( - Fig. 2).

Encouraged by the success of 5-ALA, several studies analyzed the efficacy and applicability of the comparably less expensive agent fluorescein for resection of malignant gliomas, indicating a propensity for improving EOR (-Table 2). ${ }^{51,54,56-59}$ However, many of these studies are retrospective, and none of these studies are randomized and may be confounded by case selection.

Several groups reported GTR rates of $80 \%$ using the YELLOW 560 filter. ${ }^{54,56}$ Diaz et al reported GTR in $100 \%$ when using fluorescein in their cohort of 12 glioblastoma patients and demonstrated a good correlation between intraoperative fluorescence and contrast enhancement on MRI. ${ }^{51}$ However, the authors emphasized that the accumulation of fluorescein in malignant glioma is related to the passage through the disrupted BBB and cannot be attributed to a specific uptake by the tumor itself, as is the case for 5-ALA. ${ }^{51,56}$

In addition, an analysis of the literature shows no clear consensus about dosage and timing of administration of fluorescein before surgery, although timing seems to be critical because extravasation and distribution of fluorescein follow a certain time course. Intravascular fluorescein will be extravasated after a half-life of 264 minutes and might stain edema in peritumoral normal brain parenchyma as well, increasing the danger of resection of nontumorous tissue. ${ }^{60}$ Timing of administration should be planned carefully to minimize these confounders. Furthermore, surgical manipulation of brain tissue will per se disrupt the BBB, leading to unselective extravasation of fluorescein from the bloodstream along the cutting margins, also jeopardizing confident delineation between tumor and normal tissue. Therefore, fluorescein is rather a marker of BBB integrity than a specific tumor-targeting tool. ${ }^{61}$ This aspect has to be kept in mind when using this agent.

So far, no studies have revealed reliable data on the effects of fluorescein-guided resection on outcome and survival. Two prospective controlled studies evaluated the effect on survival. One small study described an improved PFS when using fluorescein (7.2 months versus 5.4 months; $p=0.033$ ). However, the study lacked randomization and did not use special microscope filters to visualize fluorescence, using only white light. ${ }^{57,59} \mathrm{~A}$ phase II trial (FLUGLIO) evaluated the safety and efficacy of fluorescein in glioma surgery and showed that fluorescein is feasible and safe, allowing complete tumor resection in a high percentage of cases. ${ }^{62}$ Nevertheless, further prospective randomized controlled studies are warranted to investigate the benefit of fluorescein for EOR and outcome in glioma patients.

The simultaneous use of 5-ALA and fluorescein was shown to be feasible in glioblastoma surgery. 5-ALA was used to stain the tumor and fluorescein to provide tissue fluorescence of adjacent brain, leading to highly specific tumor visualization as well as enhanced background brightness at the same time. ${ }^{63}$

\section{Indocyanine Green}

ICG is a tricarbocyanine with fluorescence in the near-infrared range (NIR) and was approved by the FDA in 1959 for the diagnosis of liver function. ICG has been widely used in ophthalmology. It has a peak emission at $780 \mathrm{~nm}$ and excitation at $810 \mathrm{~nm} .{ }^{64,65}$ ICG is considered safe with a low incidence of adverse side effects such as hypotension, arrhythmia, and anaphylactic shock in $0.05 \%$, and mild symptoms such as nausea or skin eruptions in $0.2 \%{ }^{29}$

The use of ICG in neurosurgery was first described by Raabe et al for visualization of blood flow in cerebral vessels under the surgical microscope, and it is now a frequently used technique in the surgery of aneurysms and other vascular malformations. ${ }^{29,66,67}$

Recently, ICG was used for visualization of malignant gliomas using a technique referred to as second window ICG (SWIG). Twenty-four hours before surgery, $5 \mathrm{mg} / \mathrm{kg}$ BW ICG are administered to the patient, leading to the accumulation in tumor tissue mainly due to enhanced permeability and retention effects. ${ }^{68,69}$ A NIR camera (NIR light range: $700-850 \mathrm{~nm}$ ), integrated into the surgical microscope, is used to visualize the tumor at an emission of 780 to $950 \mathrm{~nm}$.

Compared with 5-ALA and fluorescein, which both emit fluorescence within the visible spectrum, ICG has excitation and emission in the NIR region of the spectrum. This advantage enables visualization of ICG fluorescence even in deeper regions, up to $3 \mathrm{~cm}$, and also through the dura. This circumstance helps in planning a precise durotomy and corticotomy. ${ }^{70}$

A pilot study evaluating SWIG in 15 patients with gliomas revealed strong tumor-to-background fluorescence ratios, and a good correlation of contrast enhancement on MRI with intraoperative fluorescence. However, the specificity was very low, $45 \%$, indicating possible illumination of adjacent edema. ${ }^{68}$ Up to now, no studies have evaluated the benefit of ICG regarding improvement of EOR or outcome in treatment of gliomas, and further research is warranted to assess the usefulness of ICG.

\section{Novel Techniques for Targeting Glioma Cells}

Fluorescence-guided surgery has to date been widely implemented in the daily routine for glioblastoma surgery. However, in its present form there are some limitations regarding the sensitivity for visualization of tumor cells. Consequently, these techniques are being further improved, and other methods, some of them still in the fledgling stages, are undergoing intense research.

\section{Tumor-Targeting Alkylphosphocholine Analogs}

Alkylphosphocholine analogs (APCs) are small synthetic phospholipid ether molecules with a purported broad tumor-targeting potential because they are known to be 


\begin{tabular}{|c|c|c|c|c|c|c|c|c|}
\hline ¿ & 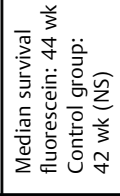 & 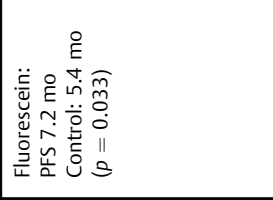 & $\frac{\alpha}{z}$ & $\stackrel{o}{z}$ & $\frac{\alpha}{z}$ & $\frac{\alpha}{z}$ & $\frac{\alpha}{2}$ & 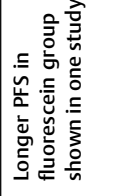 \\
\hline $\overrightarrow{\frac{a}{2}}$ & $\ddot{z}$ & $\stackrel{o v}{z}$ & $\stackrel{o r}{z}$ & $\stackrel{x}{z}$ & $\frac{\alpha}{z}$ & $\stackrel{o x}{z}$ & $\frac{x}{z}$ & $\underline{x}$ \\
\hline à & $\frac{\alpha}{z}$ & $\stackrel{\alpha}{z}$ & $\stackrel{o x}{z}$ & $\stackrel{\alpha}{z}$ & $\stackrel{\not}{z}$ & $\frac{\mathscr{L}}{z}$ & $\underline{z}$ & $\frac{n}{2}$ \\
\hline 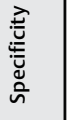 & $\frac{\alpha}{z}$ & $\frac{\alpha}{z}$ & $\frac{\alpha}{z}$ & $\begin{array}{l}\text { in } \\
\text { i̊ } \\
\infty\end{array}$ & $\begin{array}{l}\text { sे } \\
\text { के }\end{array}$ & 乡̊ & $\underline{x}$ & 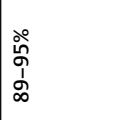 \\
\hline 雍 & $\frac{o}{z}$ & $\stackrel{o}{z}$ & $\frac{\alpha}{z}$ & ஓें & $\underset{\infty}{\stackrel{\infty}{\sim}}$ & 高 & $\frac{\alpha}{z}$ & $\begin{array}{l}\text { なें } \\
\hat{\alpha} \\
\grave{\alpha}\end{array}$ \\
\hline ث̃ & 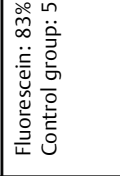 & 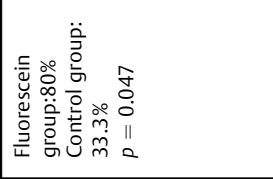 & 高 & 今̊ & 总 & 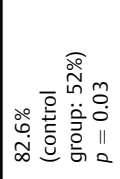 & 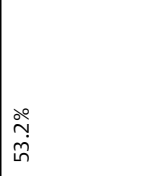 & 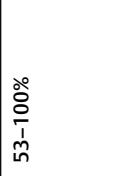 \\
\hline 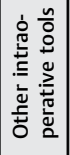 & $\frac{a}{2}$ & $\stackrel{o}{z}$ & $\frac{\infty}{z}$ & 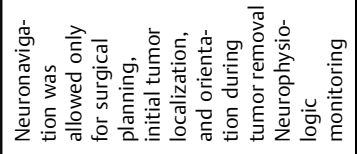 & 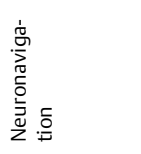 & 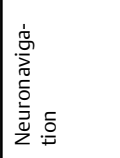 & 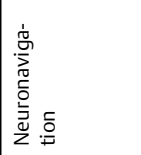 & 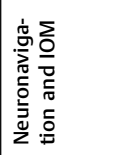 \\
\hline 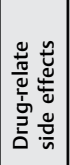 & $\frac{x}{2}$ & 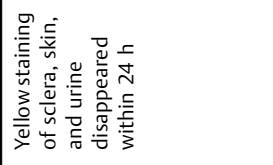 & 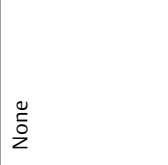 & 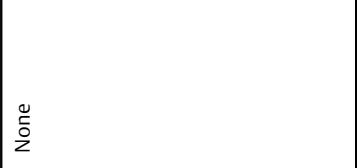 & $\stackrel{\alpha}{z}$ & \begin{tabular}{|l}
0 \\
$\frac{0}{2}$ \\
2
\end{tabular} & 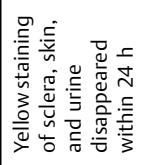 & 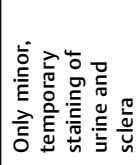 \\
\hline 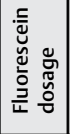 & 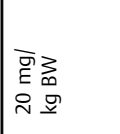 & 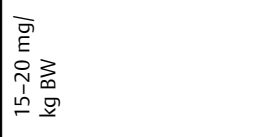 & 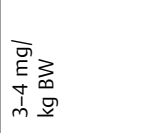 & 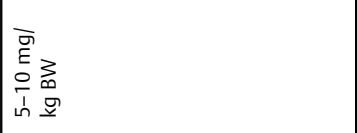 & 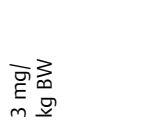 & 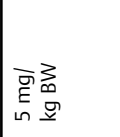 & 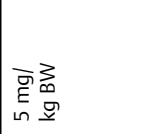 & 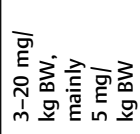 \\
\hline 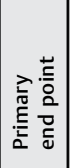 & 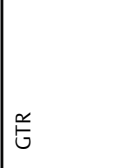 & 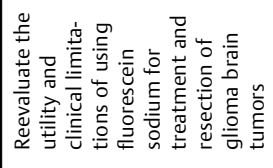 & 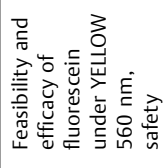 & 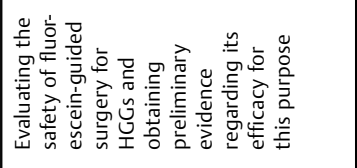 & 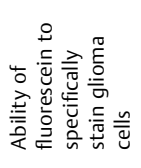 & 兰 & 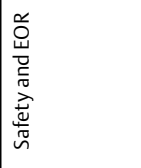 & 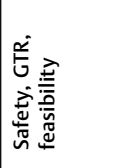 \\
\hline 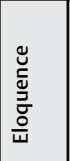 & $\frac{o}{z}$ & 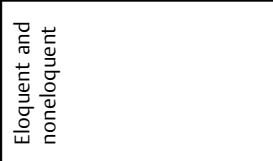 & 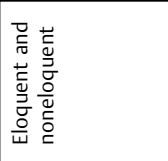 & 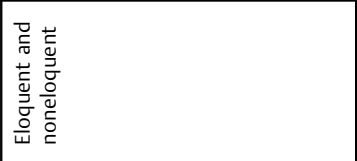 & 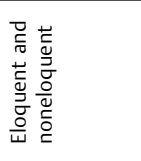 & 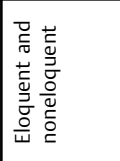 & 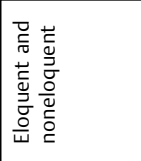 & 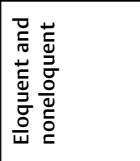 \\
\hline 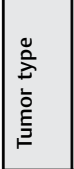 & 站 & 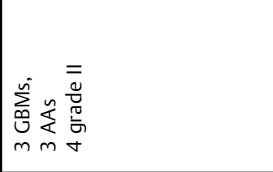 & 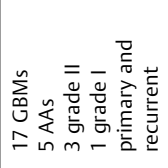 & 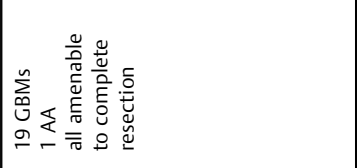 & 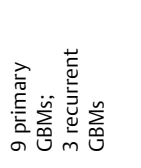 & 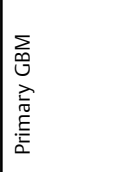 & 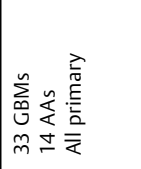 & 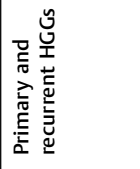 \\
\hline 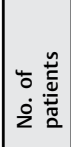 & 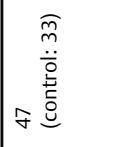 & 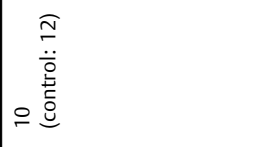 & $\stackrel{\sim}{N}$ & $\stackrel{\sim}{\sim}$ & $\simeq$ & 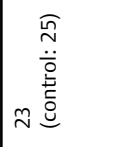 & f & $\stackrel{\llcorner}{\infty}$ \\
\hline 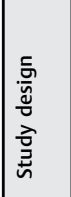 & 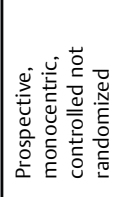 & 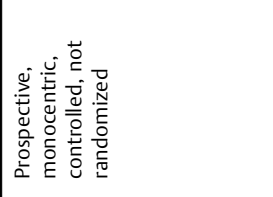 & 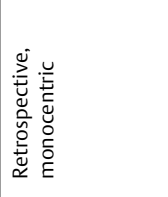 & 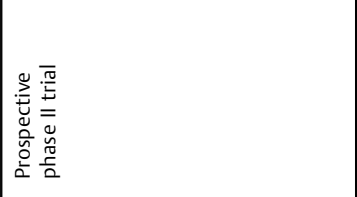 & 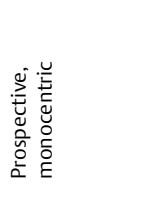 & 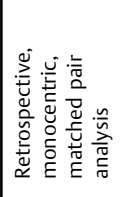 & 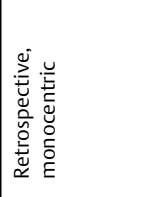 & 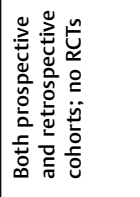 \\
\hline 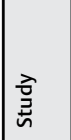 & 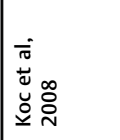 & 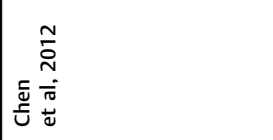 & 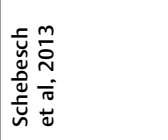 & 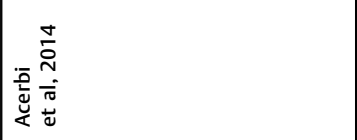 & 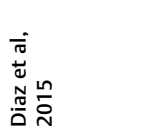 & 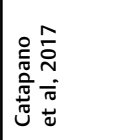 & 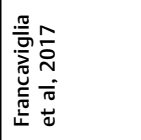 & 离 \\
\hline
\end{tabular}


taken up by malignant cells thorough overexpressed lipid rafts. Due to decreased catabolism in cancer cells, APCs undergo prolonged retention. ${ }^{71,72}$ In a glioblastoma xenograft mouse model, Swanson et al showed that two fluorescent APCs (CLR1501 green fluorescence and CLR1502 near-infrared fluorescence) are capable of labeling glioblastoma cells with high tumor-to-normal parenchyma. ${ }^{69}$ Further research aims at developing dual-labeled APCs enabling fluorescence-guided visualization and PET imaging with the same agent. Despite still being under investigation in a preclinical status, this technique offers the possibility of targeting and treating glioblastoma at different phases of the disease: resection, staging, and possibly localized radiotherapy. ${ }^{11,73}$

\section{BLZ-100 Fluorescence-Guided Brain Tumor Surgery}

BLZ-100 (tozuleristide) consists of the tumor-targeting peptide chlorotoxin, extracted from the venom of scorpions, with assumed specific binding to gliomas, conjugated with the near-infrared fluorophore ICG. ${ }^{9,74}$ The agent is administered 24 hours before surgery, and fluorescence is visualized using a NIR camera. Butte et al demonstrated a high affinity of BLZ-100 toward glioblastomas in a mouse model. ${ }^{75}$ Further studies are needed to determine the role of this technique as a further step toward using fluorescent-labeled probes with tumor-specific molecular targets to visualize glioma cells with higher accuracy in the clinical setting of glioblastoma surgery, and early-phase clinical studies are underway.

\section{Confocal Endomicroscopy}

Major limitations of FGS are the lack of high resolution and the subjective interpretation of fluorescence qualities. Especially at the tumor margin, delineation of tumor tissue from normal brain is often challenging, and prediction of histologic tumor grading from preoperative imaging is often not possible. Intraoperative frozen sections are frequently performed to acquire immediate diagnosis. However, this procedure is time consuming and can be nondiagnostic or even misleading in certain cases. ${ }^{76}$ Confocal endomicroscopy is a technique that was recently introduced into the field of neurosurgery. Images are acquired using a handheld probe that has a single optical fiber for illumination and detection. The images are displayed in high resolution in up to 1,000 -fold magnification to an LCD workstation. To provide tissue contrast, fluorescent agents like fluorescein are administered. ${ }^{10,77,78}$ Confocal endomicroscopy allows real-time visualization of malignant cells and is particularly useful for scanning the tumor margin for residual tumor tissue with high accuracy to enhance EOR and at the same time lower the risk of resection of nontumorous tissue in eloquent areas leading to possible neurologic deficits. For interpretation of acquired images, profound histopathologic knowledge or the presence of a neuropathologist is required. ${ }^{79}$

\section{Raman Spectroscopy}

Raman spectroscopy is based on the Raman effect, first described by C.V. Raman in 1928, and refers to the scattering of monochromatic light in tissue. Most photons in the visible spectrum are scattered elastically, implying they have the same level of energy when interacting with a tissue or object. However, some photons transfer or absorb energy to or from the object being imaged, resulting in a transmission of energy. This phenomenon is called inelastic scattering and known as the Raman effect. ${ }^{80}$ With the help of a spectrometer (Raman spectroscopy), information regarding the chemical composition of different tissues, for example, the amount and ratios of lipid and protein, can be obtained. These data provide a unique biochemical signature of the tissue and enable delineation between different tissues. In comparison with other techniques, Raman spectroscopy is a label-free visualization method that depends on intrinsic biochemical properties of different tissues to provide image contrast. $^{81}$ This technique was shown to be effective in delineation of glioblastoma, necrosis, and normal brain parenchyma as well. ${ }^{8,82}$ Normal, necrotic, and glioblastoma tissue was distinguished by Raman spectroscopy in frozen sections with $99.5 \%$ accuracy. ${ }^{8}$ Jermyn et al used a Raman spectroscopy handheld probe system intraoperatively and found an accuracy of $92 \%$ for glioma detection. ${ }^{83}$ Similar to confocal endomicroscopy, this technique enables intraoperative tissue analysis before resection and is a promising guide for surgical resection and decision making. ${ }^{83,84}$

\section{Conclusion and Future Perspective}

In summary, several intraoperative imaging methods aiming at improvement of intraoperative glioma targeting and visualization are presently available. Neurosurgeons have started to integrate these techniques into their daily routine for glioma surgery. The ultimate purpose of these methods is to increase the EOR while keeping the risk for postoperative neurologic deterioration low.

Still, there are limitations, as discussed earlier and listed in - Table 3, that have to be considered when applying one of these techniques. To overcome these limitations, further research is being performed. One approach is the combination of different techniques, such as neuronavigation and FGS, allowing the generation of comprehensive information on tumor extent, anatomy, and metabolism. Adding newer techniques, like Raman spectroscopy or targeted fluorescence, further information regarding chemical and metabolic composition of the tissue will be provided.

5-ALA appears to be the only available intraoperative tool for direct identification of glioblastoma cells. It has further shown a good correlation with regions of higher metabolic activity in tumor, similar to FET (fluoroethyltyrosine)-PET, although these PET hot spots often cannot be matched on MRI. ${ }^{85,86}$ In addition, a higher Ki-67/MIB-1 index and other features of malignancy correlate with the amount of 5-ALA fluorescence observed. ${ }^{87,88}$ Most randomized controlled trials are based on the gadolinium-based assessment of 
484 Identifying and Targeting Glioblastoma Cells during Surgery Schipmann et al.

Table 3 Overview of current techniques for intraoperative visualization of glioblastoma cells with their advantages and disadvantages

\begin{tabular}{|c|c|c|c|c|c|}
\hline Technique & Publications & Principle & $\begin{array}{l}\text { Application/ } \\
\text { development }\end{array}$ & Advantages & Disadvantages \\
\hline Neuronavigation & $\begin{array}{l}\text { Maciunas et al, } 1996 \\
\text { Jung et al, } 2006 \\
\text { Wirtz et al, } 2000 \\
\text { Orringer et al, } 2012\end{array}$ & $\begin{array}{l}\text { Preoperative } \\
\text { images, } \\
\text { intraoperative } \\
\text { orientation }\end{array}$ & $\begin{array}{l}\text { Widespread use in } \\
\text { clinical setting }\end{array}$ & $\begin{array}{l}\text { - Maintaining orientation } \\
\text { - Visualization of anatomy } \\
\text { - Planning surgical approach } \\
\text { - Combination with other tools } \\
\text { - Access to various preoperative } \\
\text { images including PET, CT, MRI, } \\
\text { data for fiber tracking }\end{array}$ & $\begin{array}{l}\text { - Brain shift, loss of accuracy } \\
\text { - Relies on preoperative } \\
\text { imaging, not real time } \\
\text { - Interruption if surgical workflow }\end{array}$ \\
\hline Ultrasound & $\begin{array}{l}\text { Mercier et al, } 2011 \\
\text { Saether et al, } 2012\end{array}$ & $\begin{array}{l}\text { Intraoperative } \\
\text { imaging }\end{array}$ & $\begin{array}{l}\text { Widespread use in } \\
\text { clinical setting }\end{array}$ & $\begin{array}{l}\text { - Dynamic, cheap, and easy to use } \\
\text { - Provides intraoperative real-time } \\
\text { images } \\
\text { - May be used to update } \\
\text { navigation system }\end{array}$ & - Low resolution \\
\hline iMRI & $\begin{array}{l}\text { Hatiboglu et al, } 2009 \\
\text { Senft et al, } 2011 \\
\text { Liang et al, } 2012 \\
\text { Ozduman et al, } 2014 \\
\text { Coburger et al, } 2014 \\
\text { Coburger et al, } 2015\end{array}$ & $\begin{array}{l}\text { Intraoperative } \\
\text { imaging }\end{array}$ & $\begin{array}{l}\text { Widespread use in } \\
\text { clinical setting }\end{array}$ & $\begin{array}{l}\text { - Almost real-time images during } \\
\text { surgery } \\
\text { - Identification of residual tumor }\end{array}$ & $\begin{array}{l}\text { - Expensive } \\
\text { - Time consuming } \\
\text { - Extends duration of surgery and } \\
\text { anesthesia }\end{array}$ \\
\hline 5-ALA & $\begin{array}{l}\text { Stummer et al, } 1998 \\
\text { Stummer et al, } 2000 \\
\text { Stummer et al, } 2006 \\
\text { Nabavi et al, } 2009 \\
\text { Díez Valle et al, } 2011 \\
\text { Della Puppa et al, } 2012 \\
\text { Schucht et al, } 2012 \\
\text { Della Puppa et al, } 2012 \\
\text { Stummer et al, } 2014 \\
\text { Schucht et al, } 2014 \\
\text { Lau et al, } 2016\end{array}$ & Metabolic & $\begin{array}{l}\text { Widespread use in } \\
\text { clinical setting, } \\
\text { FDA and } \\
\text { EMA approval }\end{array}$ & $\begin{array}{l}\text { - Selectively absorbed by tumor } \\
\text { cells } \\
\text { - Low toxicity, high safety } \\
\text { - Intraoperative real-time } \\
\text { imaging } \\
\text { - Full integration into the } \\
\text { surgical microscope and view of } \\
\text { full surgical field } \\
\text { - Use without interruption to the } \\
\text { surgical workflow } \\
\text { - Reliable correlation with } \\
\text { preoperative contrast enhance- } \\
\text { ment on MRI } \\
\text { - Correlation with histopathology } \\
\text { - Brain shift is no concern }\end{array}$ & $\begin{array}{l}\text { - Low background illumination } \\
\text { o Alternating between white light } \\
\text { and fluorescence mode } \\
\text { - Imaging surface tool, depth can } \\
\text { limit visualization } \\
\text { - Requires special microscope } \\
\text { - Expensive } \\
\text { - Bleaching effect } \\
\text { - Time dependency } \\
\text { - Subjective interpretation of } \\
\text { fluorescence intensities }\end{array}$ \\
\hline Fluorescein & $\begin{array}{l}\text { Koc et al, } 2008 \\
\text { Chen et al, } 2012 \\
\text { Schebesch et al, } 2013 \\
\text { Acerbi et al, } 2014 \\
\text { Diaz et al, } 2015 \\
\text { Francaviglia et al, } 2017 \\
\text { Catapano et al, } 2017\end{array}$ & $\begin{array}{l}\text { Permeability } \\
\text { of BBB }\end{array}$ & $\begin{array}{l}\text { Human use, } \\
\text { off-label }\end{array}$ & $\begin{array}{l}\text { - Robust, safe, cheap } \\
\text { - Can be visualized under white } \\
\text { light (using higher concentra- } \\
\text { tions) } \\
\text { - Intraoperative real-time } \\
\text { imaging } \\
\text { - Full integration into the } \\
\text { surgical microscope and view of } \\
\text { the full surgical field } \\
\text { - Use without interruption to the } \\
\text { surgical workflow } \\
\text { - Brain shift is no concern }\end{array}$ & $\begin{array}{l}\text { - Not tumor cell specific } \\
\text { o Marker of BBB breakdown } \\
\text { - Unselective extravasation } \\
\text { during surgery } \\
\text { - Time dependency } \\
\text { - Subjective interpretation of } \\
\text { fluorescence intensities }\end{array}$ \\
\hline ICG & Lee et al, 2016 & $\begin{array}{l}\text { Permeability } \\
\text { of BBB }\end{array}$ & $\begin{array}{l}\text { Human use, } \\
\text { off-label }\end{array}$ & $\begin{array}{l}\text { - Excitation and emission in the } \\
\text { near-infrared region } \\
\text { O Enables visualization of fluores- } \\
\text { cence situated deeper in the tissue } \\
\text { - Low toxicity, high safety } \\
\text { - Intraoperative real-time } \\
\text { imaging } \\
\text { - Brain shift does not interfere with } \\
\text { this technique } \\
\text { - Full integration into the } \\
\text { surgical microscope and view of the } \\
\text { full surgical field } \\
\text { - Use without interruption to the } \\
\text { surgical workflow } \\
\text { - Brain shift is no concern }\end{array}$ & $\begin{array}{l}\text { - Requires special cameras to } \\
\text { visualize fluorescence } \\
\text { - Not tumor specific } \\
\text { - Accumulates due to an enhanced } \\
\text { permeability of the BBB } \\
\text { - Time dependency } \\
\text { - Subjective interpretation of } \\
\text { fluorescence intensities }\end{array}$ \\
\hline $\begin{array}{l}\text { Tumor-targeted } \\
\text { alkylphosphocholine } \\
\text { analogs }\end{array}$ & Swanson et al, 2015 & Tumor-targeted & Animal model & - Specific detection of tumor cells & \\
\hline $\begin{array}{l}\text { BLZ-100 } \\
\text { (tozuleristide) }\end{array}$ & Butte et al, 2013 & Tumor-targeted & Animal model & - Tumor-specific molecular targets & \\
\hline $\begin{array}{l}\text { Confocal } \\
\text { endomicroscopy }\end{array}$ & $\begin{array}{l}\text { Hoffman et al, } 2006 \\
\text { Foersch et al, } 2012\end{array}$ & $\begin{array}{l}\text { Intraoperative } \\
\text { microscopy, } \\
\text { fluorescence } \\
\text { labeling }\end{array}$ & $\begin{array}{l}\text { Human use, } \\
\text { clinical trials }\end{array}$ & $\begin{array}{l}\text { - Intraoperative neuropathologic } \\
\text { diagnostic } \\
\text { - High resolution in up to } \\
1,000 \text {-fold magnification } \\
\text { - High accuracy }\end{array}$ & $\begin{array}{l}\text { - Only small field can be analyzed at } \\
\text { the same time } \\
\text { - Time consuming } \\
\text { - Presence of a neuropathologist } \\
\text { required to interpret images }\end{array}$ \\
\hline $\begin{array}{l}\text { Raman } \\
\text { spectroscopy }\end{array}$ & $\begin{array}{l}\text { Krafft et al, } 2004 \\
\text { Kalkanis et al, } 2014 \\
\text { Jermyn et al, } 2015\end{array}$ & $\begin{array}{l}\text { Intrinsic } \\
\text { biochemical } \\
\text { properties of } \\
\text { different tissues }\end{array}$ & $\begin{array}{l}\text { Human use, } \\
\text { clinical trials }\end{array}$ & $\begin{array}{l}\text { - Unique biochemical signature } \\
\text { - High accuracy }\end{array}$ & $\begin{array}{l}\text { - Only small field can be analyzed at } \\
\text { the same time } \\
\text { - Time consuming }\end{array}$ \\
\hline
\end{tabular}

Abbreviations: 5-ALA, 5-aminolevulinic acid; BBB, blood-brain barrier; CT, computed tomography; EMA, European Medicines Agency; FDA, Food and Drug Administration; ICG, indocyanine green; iMRI, intraoperative magnetic resonance imaging; MRI, magnetic resonance imaging; PET, positron emission tomography. 
residual tumor and EOR. For the future, the EOR based on 5-ALA-induced fluorescence might be a more accurate marker.

Currently, the intensity of fluorescence relies on the subjective interpretation of the surgeon. To quantify fluorescence, further attempts have been undertaken, for example, using spectroscopic techniques to determine intraoperative protoporphyrin (Pp) IX concentration in tumor tissue via a handheld device, even in cases with no visible fluorescence under the surgical microscope. ${ }^{89}$ For low-grade glioma, where fluorescence is often not visible using standard surgical microscopy, a 100 -fold increase in sensitivity of fluorescence detection using handheld spectroscopy can be achieved, resulting in detection of PpIX fluorescence in these slowly growing tumors also. ${ }^{90}$

Targeted fluorescence imaging will soon be available, together with innovations in neurosurgical microscope technology, to help detect optical features in gliomas presently invisible to the human eye. ${ }^{70}$ Such technologies will help overcome the limitations of the sensitivity and specificity of the present methods.

\section{Conflict of Interest}

None declared.

\section{References}

1 Koshy M, Villano JL, Dolecek TA, et al. Improved survival time trends for glioblastoma using the SEER 17 population-based registries. J Neurooncol 2012;107(01):207-212

2 Ostrom QT, Gittleman H, Truitt G, Boscia A, Kruchko C, BarnholtzSloan JS. CBTRUS statistical report: primary brain and other central nervous system tumors diagnosed in the United States in 2011-2015. Neuro Oncol 2018;20(04, Suppl 4):iv1-iv86

3 Stupp R, Mason WP, van den Bent MJ, et al; European Organisation for Research and Treatment of Cancer Brain Tumor and Radiotherapy Groups; National Cancer Institute of Canada Clinical Trials Group. Radiotherapy plus concomitant and adjuvant temozolomide for glioblastoma. N Engl J Med 2005;352(10):987-996

4 Lacroix M, Abi-Said D, Fourney DR, et al. A multivariate analysis of 416 patients with glioblastoma multiforme: prognosis, extent of resection, and survival. J Neurosurg 2001;95(02):190-198

5 Sanai N, Polley MY, McDermott MW, Parsa AT, Berger MS. An extent of resection threshold for newly diagnosed glioblastomas. J Neurosurg 2011;115(01):3-8

6 Stupp R, Hegi ME, Mason WP, et al; European Organisation for Research and Treatment of Cancer Brain Tumour and Radiation Oncology Groups; National Cancer Institute of Canada Clinical Trials Group. Effects of radiotherapy with concomitant and adjuvant temozolomide versus radiotherapy alone on survival in glioblastoma in a randomised phase III study: 5-year analysis of the EORTC-NCIC trial. Lancet Oncol 2009;10(05):459-466

7 Desroches J, Jermyn M, Mok K, et al. Characterization of a Raman spectroscopy probe system for intraoperative brain tissue classification. Biomed Opt Express 2015;6(07):2380-2397

8 Kalkanis SN, Kast RE, Rosenblum ML, et al. Raman spectroscopy to distinguish grey matter, necrosis, and glioblastoma multiforme in frozen tissue sections. J Neurooncol 2014;116(03):477-485

9 Lyons SA, O'Neal J, Sontheimer H. Chlorotoxin, a scorpion-derived peptide, specifically binds to gliomas and tumors of neuroectodermal origin. Glia 2002;39(02):162-173

10 Martirosyan NL, Cavalcanti DD, Eschbacher JM, et al. Use of in vivo near-infrared laser confocal endomicroscopy with indocyanine green to detect the boundary of infiltrative tumor. J Neurosurg 2011;115(06):1131-1138
11 Zhang RR, Swanson KI, Hall LT, Weichert JP, Kuo JS. Diapeutic cancer-targeting alkylphosphocholine analogs may advance management of brain malignancies. CNS Oncol 2016;5(04): 223-231

12 Maciunas RJ, Berger MS, Copeland B, Mayberg MR, Selker R, Allen GS. A technique for interactive image-guided neurosurgical intervention in primary brain tumors. Neurosurg Clin N Am 1996;7 (02):245-266

13 Jung TY, Jung S, Kim IY, et al. Application of neuronavigation system to brain tumor surgery with clinical experience of 420 cases. Minim Invasive Neurosurg 2006;49(04):210-215

14 Schulz C, Waldeck S, Mauer UM. Intraoperative image guidance in neurosurgery: development, current indications, and future trends. Radiol Res Pract 2012;2012:197364

15 Wirtz CR, Albert FK, Schwaderer M, et al. The benefit of neuronavigation for neurosurgery analyzed by its impact on glioblastoma surgery. Neurol Res 2000;22(04):354-360

16 Orringer DA, Golby A, Jolesz F. Neuronavigation in the surgical management of brain tumors: current and future trends. Expert Rev Med Devices 2012;9(05):491-500

17 Sæther CA, Torsteinsen M, Torp SH, Sundstrøm S, Unsgård G, Solheim O. Did survival improve after the implementation of intraoperative neuronavigation and 3D ultrasound in glioblastoma surgery? A retrospective analysis of 192 primary operations. J Neurol Surg A Cent Eur Neurosurg 2012;73(02):73-78

18 Rygh OM, Selbekk T, Torp SH, Lydersen S, Hernes TA, Unsgaard G. Comparison of navigated 3D ultrasound findings with histopathology in subsequent phases of glioblastoma resection. Acta Neurochir (Wien) 2008;150(10):1033-1041; discussion 1042

19 Mursch K, Scholz M, Brück W, Behnke-Mursch J. The value of intraoperative ultrasonography during the resection of relapsed irradiated malignant gliomas in the brain. Ultrasonography 2017; 36(01):60-65

20 Mercier L, Del Maestro RF, Petrecca K, et al. New prototype neuronavigation system based on preoperative imaging and intraoperative freehand ultrasound: system description and validation. Int J CARS 2011;6(04):507-522

21 Liang D, Schulder M. The role of intraoperative magnetic resonance imaging in glioma surgery. Surg Neurol Int 2012;3(Suppl 4): S320-S327

22 Jolesz FA. Intraoperative imaging in neurosurgery: where will the future take us? Acta Neurochir Suppl (Wien) 2011;109:21-25

23 Sutherland GR, Kaibara T, Louw D, Hoult DI, Tomanek B, Saunders J. A mobile high-field magnetic resonance system for neurosurgery. J Neurosurg 1999;91(05):804-813

24 Senft C, Bink A, Franz K, Vatter H, Gasser T, Seifert V. Intraoperative MRI guidance and extent of resection in glioma surgery: a randomised, controlled trial. Lancet Oncol 2011;12(11):997-1003

25 Hatiboglu MA, Weinberg JS, Suki D, et al. Impact of intraoperative high-field magnetic resonance imaging guidance on glioma surgery: a prospective volumetric analysis. Neurosurgery 2009;64 (06):1073-1081; discussion 1081

26 Özduman K, Yıldız E, Dinçer A, Sav A, Pamir MN. Using intraoperative dynamic contrast-enhanced T1-weighted MRI to identify residual tumor in glioblastoma surgery. J Neurosurg 2014;120 (01):60-66

27 Moore GE. Fluorescein as an agent in the differentiation of normal and malignant tissues. Science 1947;106(2745):130-131

28 Stummer W, Stocker S, Wagner S, et al. Intraoperative detection of malignant gliomas by 5 -aminolevulinic acid-induced porphyrin fluorescence. Neurosurgery 1998;42(03):518-525; discussion 525-526

29 Raabe A, Beck J, Gerlach R, Zimmermann M, Seifert V. Nearinfrared indocyanine green video angiography: a new method for intraoperative assessment of vascular flow. Neurosurgery 2003;52(01):132-139; discussion 139

30 Colditz MJ, Leyen Kv, Jeffree RL. Aminolevulinic acid (ALA)-protoporphyrin IX fluorescence guided tumour resection. Part 2: 
theoretical, biochemical and practical aspects. J Clin Neurosci 2012;19(12):1611-1616

31 Stummer W, Stepp H, Möller G, Ehrhardt A, Leonhard M, Reulen HJ. Technical principles for protoporphyrin-IX-fluorescence guided microsurgical resection of malignant glioma tissue. Acta Neurochir (Wien) 1998;140(10):995-1000

32 Stummer W, Stocker S, Novotny A, et al. In vitro and in vivo porphyrin accumulation by $\mathrm{C} 6$ glioma cells after exposure to 5 -aminolevulinic acid. J Photochem Photobiol B 1998;45(2-3):160-169

33 Kaneko S, Suero Molina E, Ewelt C, Warneke N, Stummer W. Fluorescence-based measurement of real-time kinetics of protoporphyrin IX after 5-Aminolevulinic acid administration in human in situ malignant gliomas. Neurosurgery. In press

34 Stummer W, Pichlmeier U, Meinel T, Wiestler OD, Zanella F, Reulen HJ; ALA-Glioma Study Group. Fluorescence-guided surgery with 5-aminolevulinic acid for resection of malignant glioma: a randomised controlled multicentre phase III trial. Lancet Oncol 2006;7(05):392-401

35 Teixidor P, Arráez MA, Villalba G, et al. Safety and efficacy of 5aminolevulinic acid for high grade glioma in usual clinical practice: a prospective cohort study. PLoS One 2016;11(02):e0149244

36 Eljamel S. 5-ALA fluorescence image guided resection of glioblastoma multiforme: a meta-analysis of the literature. Int J Mol Sci 2015;16(05):10443-10456

37 Stummer W, Novotny A, Stepp H, Goetz C, Bise K, Reulen HJ. Fluorescence-guided resection of glioblastoma multiforme by using 5-aminolevulinic acid-induced porphyrins: a prospective study in 52 consecutive patients. J Neurosurg 2000;93(06): 1003-1013

38 Stummer W, Tonn JC, Goetz C, et al. 5-Aminolevulinic acidderived tumor fluorescence: the diagnostic accuracy of visible fluorescence qualities as corroborated by spectrometry and histology and postoperative imaging. Neurosurgery 2014;74(03): 310-319; discussion 319-320

39 Díez Valle R, Tejada Solis S, Idoate Gastearena MA, García de Eulate R, Domínguez Echávarri P, Aristu Mendiroz J. Surgery guided by 5 -aminolevulinic fluorescence in glioblastoma: volumetric analysis of extent of resection in single-center experience. J Neurooncol 2011;102(01):105-113

40 Nabavi A, Thurm H, Zountsas B, et al; 5-ALA Recurrent Glioma Study Group. Five-aminolevulinic acid for fluorescence-guided resection of recurrent malignant gliomas: a phase ii study. Neurosurgery 2009;65(06):1070-1076; discussion 1076-1077

41 Lau D, Hervey-Jumper SL, Chang S, et al. A prospective Phase II clinical trial of 5-aminolevulinic acid to assess the correlation of intraoperative fluorescence intensity and degree of histologic cellularity during resection of high-grade gliomas. J Neurosurg 2016;124(05):1300-1309

42 Schucht P, Beck J, Abu-Isa J, et al. Gross total resection rates in contemporary glioblastoma surgery: results of an institutional protocol combining 5-aminolevulinic acid intraoperative fluorescence imaging and brain mapping. Neurosurgery 2012;71(05): 927-935; discussion 935-936

43 Della Puppa A, De Pellegrin S, d'Avella E, et al. 5-aminolevulinic acid (5-ALA) fluorescence guided surgery of high-grade gliomas in eloquent areas assisted by functional mapping. Our experience and review of the literature. Acta Neurochir (Wien) 2013;155 (06):965-972; discussion 972

44 Schucht P, Seidel K, Beck J, et al. Intraoperative monopolar mapping during 5-ALA-guided resections of glioblastomas adjacent to motor eloquent areas: evaluation of resection rates and neurological outcome. Neurosurg Focus 2014;37(06):E16

45 Aldave G, Tejada S, Pay E, et al. Prognostic value of residual fluorescent tissue in glioblastoma patients after gross total resection in 5-aminolevulinic Acid-guided surgery. Neurosurgery 2013;72(06):915-920; discussion 920-921

46 Schucht P, Knittel S, Slotboom J, et al. 5-ALA complete resections go beyond MR contrast enhancement: shift corrected volumetric analysis of the extent of resection in surgery for glioblastoma. Acta Neurochir (Wien) 2014;156(02):305-312; discussion 312

47 Coburger J, Hagel V, Wirtz CR, König R. Surgery for glioblastoma: impact of the combined use of 5-aminolevulinic acid and intraoperative MRI on extent of resection and survival. PLoS One 2015; 10(06): 0131872

48 Coburger J, Engelke J, Scheuerle A, et al. Tumor detection with 5aminolevulinic acid fluorescence and Gd-DTPA-enhanced intraoperative MRI at the border of contrast-enhancing lesions: a prospective study based on histopathological assessment. Neurosurg Focus 2014;36(02):E3

49 Rabb MF, Burton TC, Schatz H, Yannuzzi LA. Fluorescein angiography of the fundus: a schematic approach to interpretation. Surv Ophthalmol 1978;22(06):387-403

50 Shinoda J, Yano H, Yoshimura S, et al. Fluorescence-guided resection of glioblastoma multiforme by using high-dose fluorescein sodium. Technical note. J Neurosurg 2003;99(03):597-603

51 Diaz RJ, Dios RR, Hattab EM, et al. Study of the biodistribution of fluorescein in glioma-infiltrated mouse brain and histopathological correlation of intraoperative findings in high-grade gliomas resected under fluorescein fluorescence guidance. J Neurosurg 2015;122(06):1360-1369

52 Hamamcıoğlu MK, Akçakaya MO, Göker B, Kasımcan MO, Kırış T. The use of the YELLOW $560 \mathrm{~nm}$ surgical microscope filter for sodium fluorescein-guided resection of brain tumors: our preliminary results in a series of 28 patients. Clin Neurol Neurosurg 2016;143:39-45

53 Schebesch KM, Brawanski A, Hohenberger C, Hohne J. Fluorescein sodium-guided surgery of malignant brain tumors: history, current concepts, and future project. Turk Neurosurg 2016;26(02):185-194

54 Schebesch KM, Proescholdt M, Höhne J, et al. Sodium fluoresceinguided resection under the YELLOW $560 \mathrm{~nm}$ surgical microscope filter in malignant brain tumor surgery-a feasibility study. Acta Neurochir (Wien) 2013;155(04):693-699

55 Kuroiwa T, Kajimoto Y, Ohta T. Development of a fluorescein operative microscope for use during malignant glioma surgery: a technical note and preliminary report. Surg Neurol 1998;50(01): 41-48; discussion 48-49

56 Acerbi F, Broggi M, Eoli M, et al. Is fluorescein-guided technique able to help in resection of high-grade gliomas? Neurosurg Focus 2014;36(02):E5

57 Chen B, Wang H, Ge P, et al. Gross total resection of glioma with the intraoperative fluorescence-guidance of fluorescein sodium. Int J Med Sci 2012;9(08):708-714

58 Francaviglia N, Iacopino DG, Costantino G, et al. Fluorescein for resection of high-grade gliomas: a safety study control in a single center and review of the literature. Surg Neurol Int 2017;8:145

59 Koc K, Anik I, Cabuk B, Ceylan S. Fluorescein sodium-guided surgery in glioblastoma multiforme: a prospective evaluation. Br J Neurosurg 2008;22(01):99-103

60 Stummer W. Poor man's fluorescence? Acta Neurochir (Wien) 2015;157(08):1379-1381

61 Schwake M, Stummer W, Suero Molina EJ, Wölfer J. Simultaneous fluorescein sodium and 5-ALA in fluorescence-guided glioma surgery. Acta Neurochir (Wien) 2015;157(05):877-879

62 Acerbi F, Broggi M, Schebesch KM, et al. Fluorescein-guided surgery for resection of high-grade gliomas: a multicentric prospective phase II study (FLUOGLIO). Clin Cancer Res 2018;24(01):52-61

63 Suero Molina E, Wölfer J, Ewelt C, Ehrhardt A, Brokinkel B, Stummer W. Dual-labeling with 5-aminolevulinic acid and fluorescein for fluorescence-guided resection of high-grade gliomas: technical note. J Neurosurg 2018;128(02):399-405

64 Cherrick GR, Stein SW, Leevy CM, Davidson CS. Indocyanine green: observations on its physical properties, plasma decay, and hepatic extraction. J Clin Invest 1960;39:592-600

65 Reinhart MB, Huntington CR, Blair LJ, Heniford BT, Augenstein VA. Indocyanine green: historical context, current applications, and future considerations. Surg Innov 2016;23(02):166-175 
66 Hänggi D, Etminan N, Steiger HJ. The impact of microscopeintegrated intraoperative near-infrared indocyanine green videoangiography on surgery of arteriovenous malformations and dural arteriovenous fistulae. Neurosurgery 2010;67(04): 1094-1103; discussion 1103-1104

67 Raabe A, Nakaji P, Beck J, et al. Prospective evaluation of surgical microscope-integrated intraoperative near-infrared indocyanine green videoangiography during aneurysm surgery. J Neurosurg 2005;103(06):982-989

68 Lee JY, Thawani JP, Pierce J, et al. Intraoperative near-infrared optical imaging can localize gadolinium-enhancing gliomas during surgery. Neurosurgery 2016;79(06):856-871

69 Swanson KI, Clark PA, Zhang RR, et al. Fluorescent cancer-selective alkylphosphocholine analogs for intraoperative glioma detection. Neurosurgery 2015;76(02):115-123; discussion 123-124

70 Valdés PA, Roberts DW, Lu FK, Golby A. Optical technologies for intraoperative neurosurgical guidance. Neurosurg Focus 2016;40 (03):E8

71 Hilgard P, Klenner T, Stekar J, Unger C. Alkylphosphocholines: a new class of membrane-active anticancer agents. Cancer Chemother Pharmacol 1993;32(02):90-95

72 Weichert JP, Clark PA, Kandela IK, et al. Alkylphosphocholine analogs for broad-spectrum cancer imaging and therapy. Sci Transl Med 2014;6(240):240ra75

73 Kuo JS, Zhang RR, Pinchuk AN, Clark PA, Weichert JP. Creation of a dual-labeled cancer-targeting alkylphosphocholine analog for dual modality quantitative positron emission tomography and intraoperative tumor visualization. Neurosurgery 2016;63(01):208

74 Parrish-Novak J, Byrnes-Blake K, Lalayeva N, et al. Nonclinical profile of BLZ-100, a tumor-targeting fluorescent imaging agent. Int J Toxicol 2017;36(02):104-112

75 Butte PV, Mamelak A, Parrish-Novak J, et al. Near-infrared imaging of brain tumors using the Tumor Paint BLZ-100 to achieve nearcomplete resection of brain tumors. Neurosurg Focus 2014;36 (02):E1

76 Uematsu Y, Owai Y, Okita R, Tanaka Y, Itakura T. The usefulness and problem of intraoperative rapid diagnosis in surgical neuropathology. Brain Tumor Pathol 2007;24(02):47-52

77 Foersch S, Heimann A, Ayyad A, et al. Confocal laser endomicroscopy for diagnosis and histomorphologic imaging of brain tumors in vivo. PLoS One 2012;7(07):e41760

78 Hoffman A, Goetz M, Vieth M, Galle PR, Neurath MF, Kiesslich R. Confocal laser endomicroscopy: technical status and current indications. Endoscopy 2006;38(12):1275-1283
79 Belykh E, Martirosyan NL, Yagmurlu K, et al. Intraoperative fluorescence imaging for personalized brain tumor resection: current state and future directions. Front Surg 2016;3:55

80 Raman C. A new type of secondary radiation. Nature 1928; 121:501-502

81 Hollon T, Lewis S, Freudiger CW, Sunney Xie X, Orringer DA. Improving the accuracy of brain tumor surgery via Raman-based technology. Neurosurg Focus 2016;40(03):E9

82 Krafft C, Neudert L, Simat T, Salzer R. Near infrared Raman spectra of human brain lipids. Spectrochim Acta A Mol Biomol Spectrosc 2005;61(07):1529-1535

83 Jermyn M, Mok K, Mercier J, et al. Intraoperative brain cancer detection with Raman spectroscopy in humans. Sci Transl Med 2015;7(274):274ra19

84 Desroches J, Jermyn M, Pinto M, et al. A new method using Raman spectroscopy for in vivo targeted brain cancer tissue biopsy. Sci Rep 2018;8(01):1792

85 Arbizu J, Tejada S, Marti-Climent JM, et al. Quantitative volumetric analysis of gliomas with sequential MRI and ${ }^{11} \mathrm{C}$-methionine PET assessment: patterns of integration in therapy planning. Eur J Nucl Med Mol Imaging 2012;39(05):771-781

86 Jaber M, Wölfer J, Ewelt C, et al. The value of 5-aminolevulinic acid in low-grade gliomas and high-grade gliomas lacking glioblastoma imaging features: an analysis based on fluorescence, magnetic resonance imaging, $18 \mathrm{~F}$-fluoroethyl tyrosine positron emission tomography, and tumor molecular factors. Neurosurgery 2016;78(03):401-411; discussion 411

87 Idoate MA, Díez Valle R, Echeveste J, Tejada S. Pathological characterization of the glioblastoma border as shown during surgery using 5-aminolevulinic acid-induced fluorescence. Neuropathology 2011;31(06):575-582

88 Roberts DW, Valdés PA, Harris BT, et al. Coregistered fluorescence-enhanced tumor resection of malignant glioma: relationships between $\delta$-aminolevulinic acid-induced protoporphyrin IX fluorescence, magnetic resonance imaging enhancement, and neuropathological parameters. Clinical article. J Neurosurg 2011;114(03):595-603

89 Valdés PA, Leblond F, Kim A, et al. Quantitative fluorescence in intracranial tumor: implications for ALA-induced PpIX as an intraoperative biomarker. J Neurosurg 2011;115(01):11-17

90 Valdés PA, Jacobs V, Harris BT, et al. Quantitative fluorescence using 5-aminolevulinic acid-induced protoporphyrin IX biomarker as a surgical adjunct in low-grade glioma surgery. J Neurosurg 2015;123(03):771-780 